\title{
BIOMIMETIC ENAMEL REMINERALIZATION USING CHITOSAN HYDROGEL (AN IN VITRO STUDY)
}

\author{
Ingy E. Ibrahim ${ }^{1} B D S$, Sahar S. Karam² PhD, Hanaa M. Aly $P h D$
}

\begin{abstract}
INTRODUCTION: Biomimetic enamel reconstruction is a significant topic in material science and dentistry as a novel approach for the treatment of dental caries or erosion. Compared to other conventional treatments, biocompatible and biodegradable chitosan hydrogel, with its antimicrobial properties shows promise as a biomaterial for the prevention, restoration, and treatment of defective enamel.

OBJECTIVES: The present study was designed to investigate the effect of chitosan on the remineralization of enamel.

MATERIALS AND METHODS Sound human premolars extracted for orthodontic reasons and were sectioned into $1 \mathrm{~mm}$ thick slices.30 slices were selected and randomly divided into 3 equal groups (10 slices per group) as follows:

GroupI: Control group, the slices were etched with $37 \%$ phosphoric acid for 30 seconds.

Group II: The slices were etched with 37\% phosphoric acid for 30 seconds, coated with bonding agent and chitosan hydrogel. Then slices were incubated at $37^{\circ} \mathrm{C}$ in artificial saliva solution for 5 days.

Group III: The slices were etched with 37\% phosphoric acid for 30 seconds, coated with bonding agent and chitosan hydrogel. Then slices were incubated at $37^{\circ} \mathrm{C}$ in artificial saliva solution for 10 days.

After remineralization, the enamel surfaces were ultrasonically cleaned for 2 minutes and analyzed with scanning electron microscope (SEM) in order to observe induced structural changes on the enamel surface then elemental analysis and $\mathrm{Ca} / \mathrm{P}$ ratios were carried out using Energy Dispersive Analysis X-Ray (EDAX) spectrometer. The obtained data were statistically analyzed.

RESULTS: Morphological changes on the enamel surface after application of chitosan observed by SEM revealed the regeneration of an enamel-like layer in both treated samples. There was a statistically significant difference between the $\mathrm{Ca} / \mathrm{P}$ ratios of the treatment groups compared to that of the control group.

CONCLUSIONS: The ultrastructural and elemental alterations noticed in this study substantiate the use of chitosan in cases of dental erosions. KEYWORDS: Enamel, remineralization, chitosan.
\end{abstract}

1. DBS - Faculty of Dentistry - Alexandria University.

2. Professor and Head of Oral Biology Department - Faculty of Dentistry - Alexandria University, Egypt.

3. Professor of Oral Biology - Faculty of Dentistry - Alexandria University

Corresponding author:

E-mail:dr_ingy_elsaid@hotmail.com

\section{INTRODUCTION}

Enamel has a unique morphological structure and distinctive mechanical properties, making it different from other mineralized tissues such as the bone and dentin in the human body. It is composed of 95-97\% highly organized hexagonal carbonated hydroxyapatite (HA) crystals $\mathrm{Ca}_{10}(\mathrm{PO} 4)_{6}(\mathrm{OH})_{2}$ by weight. These crystals are roughly parallel to form highly organized architectural units known as enamel rods. The unique shapes and organizations of enamel crystals determine the excellent mechanical properties of tooth enamel with increased hardness and resistance to fracture and acid erosionz. Ameloblast activity and the protein-mediated process of mineralization are crucial to achieving such precisely organized structures (1).

Mature enamel is acellular and therefore is not capable of any self-regeneration. Any damage is irreversible, as there is no biological process capable of preparing damaged enamel.

When HA is in contact with water, the following reaction occurs:

Precipitation $\leftrightarrow$ Dissolution

$\mathrm{Ca}_{10}\left(\mathrm{PO}_{4}\right)_{6}(\mathrm{OH})_{2} \leftrightarrow 10 \mathrm{Ca}_{2}++6\left(\mathrm{PO}_{4}\right)_{3}-+2 \mathrm{OH}-$

Solid $\leftrightarrow$ Solution

As long as the demineralization (mineral dissolution) and remineralization (mineral precipitation) occur at the same rate, there is a state of equilibrium and no net loss of enamel. However, when demineralization rate exceeds the rate of remineralization diffusion of calcium and phosphate ions out of enamel occurs and produces a chalky white spot lesion or opacity, and may progress to cavitation if demineralization continues $(2,3)$.

Enamel remineralisation is a well-accepted concept for repairing enamel defects in dental erosion. Many clinical and commercial products have been developed to improve enamel remineralisation, including fluoride varnish, fluoride dentifrice and amorphous calcium phosphate (ACP) $(4,5)$.

On the other hand, further studies showed that topical fluoride application is not the perfect mechanism for remineralization (6,7). Moreover, when amorphous calcium phosphate was applied, the crystals formed were loosely structured and morphologically irregular after treatment with these agents (8-10).

In recent years, treatment of early caries lesions by the application of Nano-sized HA has received considerable attention (11).

Earlier studies in this field had developed in vitro strategies to prepare Nano-sized hydroxyapatite (nHA) including the sol-gel method, mechano-chemical process, electrochemical deposition, crystallization under magnetic field, hydrothermal crystallization, etc. However, as reported in the literature, these strategies commonly require 
severe reaction conditions such as extremely high temperature, high pressure, highly acidic condition or involving harmful reagents $(12,13)$.

A most recent study suggested the wet precipitation method that is a relatively convenient way for the production of nHA. However this approach was associated with lengthy and complex processes combined with the need for expensive equipment (14).

Inspired by the molecular mechanism of organicmatrix mediated biomineralization, the regeneration of dental tissue with a biomimetic cell-free stratagem has been recently proposed.

In previous researches, many materials like carboxylterminated poly (amido amine) (PAMAM-COOH) and PAMAM-COOH alendronate conjugate (PAMAM-ALN$\mathrm{COOH})$ were proposed as templates to regenerate the HA crystal and achieved a great remineralization effect on tooth (15).

These methods have provided the potential for tooth defect repair via a self-healing mechanism, which is a much more ideal for use in dental clinics than the current traditional treatment measures. Though these methods may not be able to replace the traditional synthetic materials in treating large tooth defects, it may be potential to be used to treat micro tooth defect (such as the early caries, white spot lesions, dentin hypersensitivity) at the current stage. However, it is still necessary to develop a material which has both a prominent remineralization effect and antimicrobial ability (16).

Chitin, a naturally abundant polysaccharide, and the supporting material of crustaceans, insects, etc., is well known to consist of 2-acetamido-2-deoxy-b-D-glucose through a $\beta(1,4)$ linkage. Chitosan is the N-deacetylated derivative of chitin, is soluble in dilute acids such as acetic acid, formic acid, etc. Chitosan has been found wide application in biomedical fields as a result of its biocompatibility and non-toxicity, such as drug delivery, wound dressing, as a tissue replacement material, etc $(17,18)$.

Furthermore, chitosan hydrogel obtained through the polymer dilution in acetic acid has been suggested as a preventive and therapeutic material for dental caries thanks to its antimicrobial properties (19-21). The null hypothesis of this study is that chitosan may or may not provide a promising material for the biomimetic regeneration of dental hard tissues.

\section{MATERIALS AND METHODS}

This study was conducted after the approval of the Research Ethics Committee, Faculty of Dentistry Alexandria University.

Sound human premolars freshly extracted for orthodontic reasons were selected for the study excluding any teeth with deformities or cracks.

The selected teeth were thoroughly cleaned of debris and any organic material on their surfaces by immersion in $10 \%$ solution of sodium hypochlorite $(\mathrm{NaOCl})$. Then the teeth were washed with a soft brush and water and were then stored in saline $(0.9 \%$ sodium chloride solution) until required.

The human premolars were sectioned into $1 \mathrm{~mm}$ thick slices and were randomly assigned to 3 equal groups (10 slices each) as follows:

\section{Group I: Etched control group}

The slices were etched with $37 \%$ phosphoric acid for 30 seconds, ultrasonically cleaned.

\section{Group II: 5 days treated group}

The slices were etched with $37 \%$ phosphoric acid for 30 seconds, coated with chitosan hydrogel, and then immersed in artificial saliva solution for 5 days before evaluation.

\section{Group III: 10 days treated group}

The slices were etched with $37 \%$ phosphoric acid for 30 seconds, coated with chitosan hydrogel, and then immersed in artificial saliva solution for 10 days before evaluation.

\section{Preparation of acid etched tooth sections}

All the teeth were mounted in acrylic molds exposing only the crowns.

The crowns of the teeth were manually sectioned perpendicular to their long axis by means of a water-cooled low-speed diamond saw. Slices of $1 \mathrm{~mm}$ thick were cut in bucco-lingual direction. Slices obtained from the same positions along the long axis of teeth were used for all the groups in the study.

The selected slices were ultrasonically cleaned for 2 minutes, and then rinsed with deionized water 3 times.

To simulate the demineralization lesions, slices were etched using 37\% phosphoric acid for 30 seconds then immediately rinsed with deionized water and ultrasonically cleaned $(22,23)$.

The samples from group II and group III were daubed with phosphate ions dental adhesive agent (Prime \& Bond $®$ NT, Dentsply Detrey Gmbh) before the application of chitosan hydrogel. The solidification process was induced by light curing for 10 seconds at least (24).

\section{Preparation of chitosan hydrogel}

0.25gram chitosan powder (purchased from Sigma-Aldrich, Germany) was dissolved in $25 \mathrm{ml}$ acetic acid solution followed by stirring at $80{ }^{\circ} \mathrm{C}$ overnight. The chitosan solution was left to cool at room temperature. The $\mathrm{pH}$ of the chitosan solution was then adjusted by adding $1 \mathrm{M} \mathrm{NaOH}$ solution drop by drop until $\mathrm{pH}$ reached 6.5 where chitosan gel was formed (25).

\section{Application of chitosan hydrogel to treatment groups (II} \& III)

Hydrogel was applied to the etched tooth slice using a syringe and were allowed to dry at room temperature for 2 hours.

The tooth slices were transferred to beakers each containing $30 \mathrm{ml}$ of artificial saliva solution (ASS) prepared in Institute of Graduate studies and Research.

The beakers were covered with aluminum foil, and then kept in an incubator at $37^{\circ} \mathrm{C}$ for 5 days for group II and 10 days for group III (24). The solution was refreshed each day during the incubation period.

\section{Assessment of Regenerated Enamel}

The slices were ultrasonically cleaned for 2 minutes, air dried, sputter-coated with gold and the morphology of the precipitated grown out layer on the occlusal surface were characterized by a scanning electron microscope (SEM) and then elemental analysis and $\mathrm{Ca} / \mathrm{P}$ ratios were carried out using Energy Dispersive Analysis X-Ray (EDAX) spectrometer. The obtained data were statistically analyzed using ANOVA test. 


\section{RESULTS}

\section{A. Scanning Electron Microscopic Results}

The SEM examination was focused on evaluating the topographic changes of the surfaces of all the study group

\section{Group I (Control group)}

SEM images of acid etched enamel surfaces revealed loss of surface integrity resembling enamel erosive lesions. The surface was irregular with a number of porous defects suggestive of demineralization. (Fig.1A)

Two distinct patterns of demineralization were observed, the first being with relatively intact enamel rods core with a preferential loss of rods peripheries.However, etching resulted in wide inter-rod spaces and irregular flat rod tops in some areas, compromising the smooth rounded shape of natural enamel rods.(Fig. 2A)

The second demineralization pattern observed revealed a loss of enamel rod core with relatively intact peripheries yielding a microporous surface. The parallel bundles of hydroxyapatite crystals making up the inter-rod enamel were evident. Crystals were fragmented with large spaces between bundles (Fig.3A)

2. Group II (5 days incubation with biomimetic chitosan hydrogel)

Regenerated crystals uniformly precipitated on the preexisting enamel crystals. Decrease in the surface irregularities and filling of some porosities were evident compared to the control group. (Fig.1B)

The inter-rod spaces were reduced and rod shaped hydroxyapatite crystals were deposited in regular parallel bundles following the natural orientation of native enamel crystals and restored smooth rounded end rods. (Fig.2B)

Moreover, it was clear that nano-rod crystals were growing into tightly packed bundles, similar to the original enamel crystals in both appearance and size. The arrangement of crystal bundles was compacted reducing the spaces found in control group. (Fig.3B)

Meanwhile, the profile of acid-etched tooth enamel was still clearly visible although the original acid-etched surface was almost covered by growing crystals.

\section{Group III (10 days incubation with biomimetic chitosan} hydrogel):

Samples of this group showed a superior and uniform reestablishment of surface integrity in contrast to other groups.

An outstanding decrease in the porosities and surface irregularities was an important feature in this group. The regenerated crystals on the enamel surface formed a homogeneous and dense layer of mineralized tissue. (Fig.1C)

The regular growth of bundles of well-organized crystals was obvious in this group leading to the formation of a continuous compact enamel-like layer and eliminating almost all the micro-porosities and the exaggerated interrod spaces present in control group. Generally, the long axes of the crystals were radially perpendicular to the underlying enamel surface and formed bundles of enamel rod-like structures. (Fig.2C)

The high magnification image demonstrated a strong attraction between adjacent nano-rod crystals that caused them to fuse together to form an extensive layer of wellaligned bundles on the enamel surface. (Fig.3C)
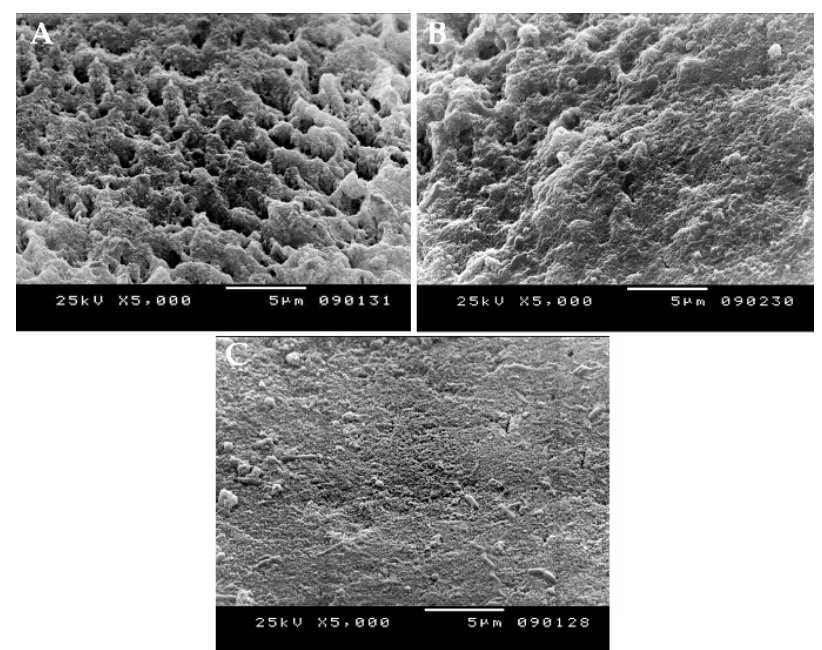

Figure (1): A) SEM of group I (control group) showing surface irregularities and porosities simulating erosive lesions(X5,000). B) SEM of group II (5 days incubation with chitosan hydrogel) showing relative decrease in surface irregularities and filling of some porosities(X5,000). C) SEM of group III (10 days incubation with chitosan hydrogel): showing a homogeneous and dense layer of mineralized tissue formed by the regenerated crystals on the enamel surface $(\mathrm{X} 5,000)$.
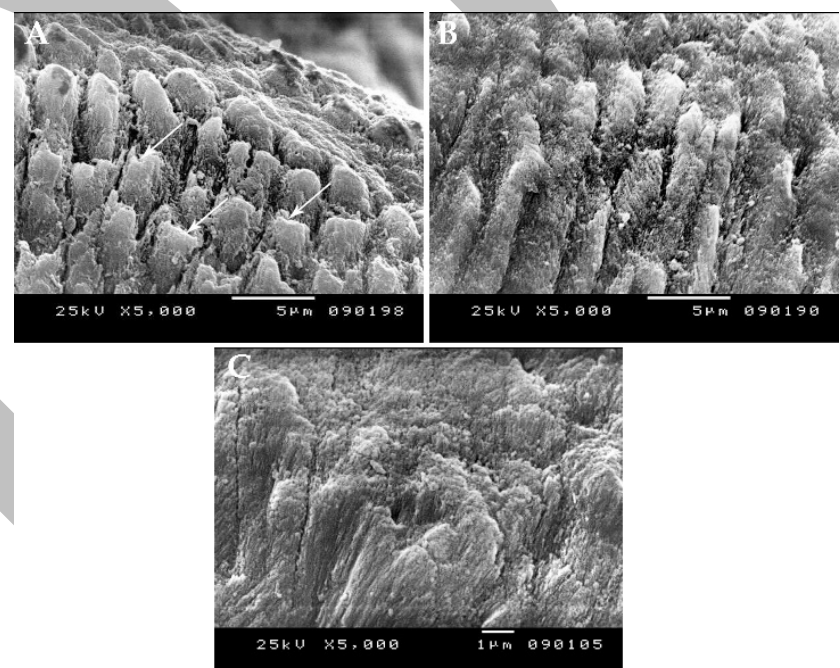

Figure (2): A) SEM of group I (control group) showing the first demineralization pattern; enamel rods are relatively intact with a preferential loss of rod peripheries. Note the tops of enamel rods are flat and irregular (white arrows) in contrast with the un-etched smooth rounded enamel rods (black arrows)(X5,000). B) SEM of group II (5 days incubation with chitosan hydrogel) showing reduced inter-rod spaces, parallel bundles of deposited crystals are formed restoring the smooth rounded end rods(X5,000). C) SEM of group III (10 days incubation with chitosan hydrogel) showing the elimination of almost all of the inter-rod spaces, bundles of crystals forms enamel rod-like structures radially perpendicular to the enamel surface $(\mathrm{X} 5,000)$. 


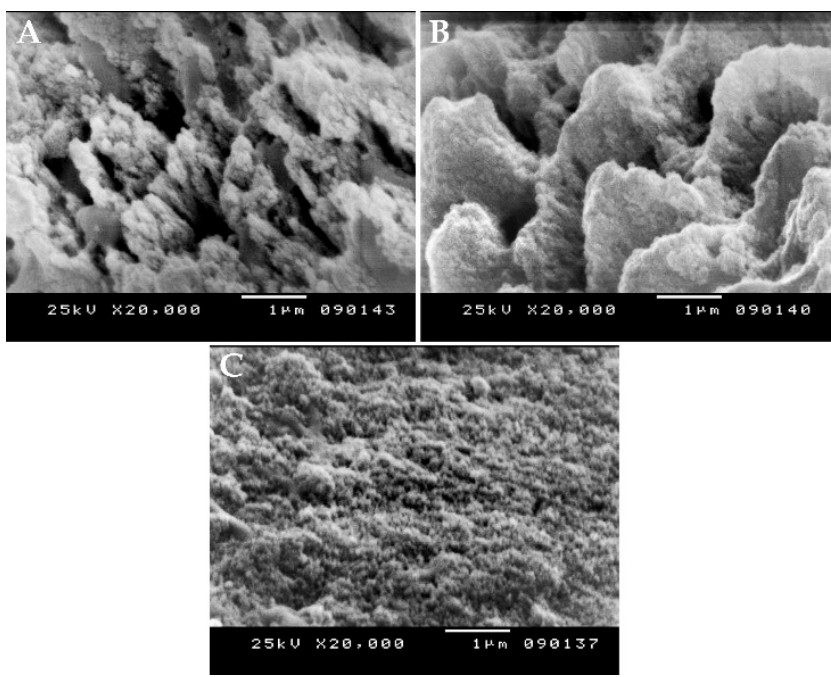

Figure (3): A) SEM of group I (control group) showing a second demineralization pattern; loss of rod core and spacing between crystal bundles. Note the inter-rod enamel formed of parallel bundles of hydroxyapatite crystals (X20,000). B) SEM of group II (5 days incubation with chitosan hydrogel) showing that regenerated crystals forms tightly packed bundles similar to original enamel bundles in appearance and size, eliminating the spaces caused by etching (arrows)(X20,000). C) SEM of group III (10 days incubation with chitosan hydrogel) showing an extensive layer of well aligned bundles of adjacent tightly packed nano-rod crystals on the enamel surface $(\mathrm{X} 20,000)$.

\section{B. Energy Dispersive Analysis X-Ray Results}

The $\mathrm{Ca}$ and $\mathrm{P}$ content (weight \%) of demineralized and remineralized enamel was measured and converted into $\mathrm{Ca} / \mathrm{P}$ ratios for each group, as demonstrated in table (1).

Table (1): Percentage values of Ca and P obtained by $\mathrm{EDAX}$ and $\mathrm{Ca} / \mathrm{P}$ ratios calculated among the studied groups.

\begin{tabular}{|c|c|c|c|}
\hline & Сa\% & P \% & $\mathrm{Ca} / \mathbf{P}$ \\
\hline \multirow{7}{*}{$\begin{array}{l}\text { Group I } \\
\text { Acid etched } \\
\text { control group }\end{array}$} & 58.0 & 42.0 & 1.38 \\
\hline & 54.5 & 45.5 & 1.19 \\
\hline & 50.7 & 49.3 & 1.02 \\
\hline & 52.3 & 47.7 & 1.09 \\
\hline & 51.1 & 48.9 & 1.04 \\
\hline & 50.2 & 49.8 & 1.008 \\
\hline & 50.1 & 49.9 & 1.004 \\
\hline \multirow{7}{*}{$\begin{array}{l}\text { Group II } \\
5 \text { days } \\
\text { incubation } \\
\text { with chitosan }\end{array}$} & 60.9 & 39.1 & 1.55 \\
\hline & 60.5 & 39.5 & 1.53 \\
\hline & 61.0 & 39.0 & 1.56 \\
\hline & 60.03 & 39.97 & 1.50 \\
\hline & 61.1 & 38.9 & 1.53 \\
\hline & 60.7 & 39.3 & 1.54 \\
\hline & 60.4 & 39.6 & 1.52 \\
\hline \multirow{4}{*}{$\begin{array}{l}\text { Group III } \\
10 \text { days } \\
\text { incubation } \\
\text { with chitosan }\end{array}$} & 62.3 & 37.7 & 1.65 \\
\hline & 63.4 & 36.6 & 1.73 \\
\hline & 62.7 & 37.3 & 1.68 \\
\hline & 62.5 & 37.5 & 1.66 \\
\hline
\end{tabular}

\begin{tabular}{|c|c|c|c|}
\hline \multirow{2}{*}{67.38} & 32.62 & 2.06 \\
\cline { 2 - 4 } & 65.02 & 34.98 & 1.85 \\
\hline & 62.0 & 38.0 & 1.63 \\
\hline
\end{tabular}

The mean values of $\mathrm{Ca} / \mathrm{P}$ ratio for each group were calculated and statistically analyzed using ANOVA test as follows:

The results revealed significant mean difference in the calcium phosphorus ratio among the three groups. The mean $\mathrm{Ca} / \mathrm{P}$ ratio was $1.12,1.55$, and 1.65 among Group I, Group II and Group III respectively. ( $F=33.12, p=0.01)$. Post-hoc pairwise comparison depicted a significant difference in the intervention groups compared to the control group, as indicated in table (2).

Table (2): Comparative tabulation of the mean $\mathrm{Ca} / \mathrm{P}$ ratios among the studied groups using ANOVA test.

\begin{tabular}{|l|l|l|c|}
\hline & $\begin{array}{l}\text { GROUP I } \\
\text { Acid etched } \\
\text { control group }\end{array}$ & $\begin{array}{l}\text { GROUP II } \\
\text { 5 days } \\
\text { incubation with } \\
\text { chitosan }\end{array}$ & $\begin{array}{l}\text { GROUP III } \\
\text { incubation with } \\
\text { chitosan }\end{array}$ \\
\hline Mean Ca/P ratio & $1.12 \pm 0.12$ & $1.55 \pm 0.09$ & $1.65 \pm 0.01$ \\
\hline P1 & & $0.01^{*}$ & $0.03^{*}$ \\
\hline P2 & $0.01^{*}$ & & 0.09 \\
\hline P3 & $0.03^{*}$ & 0.09 & \\
\hline
\end{tabular}

*P value using One-Way ANOVA test was considered significant $(\mathrm{F}=33.12$, overall $\mathrm{p}=0.01)$

*P1, P2, P3: Post-hoc pairwise comparison between the different groups

\section{DISCUSSION}

One alternative strategy to overcome the challenges of conventional treatments of initial enamel lesions is to regrow an enamel-like layer directly onto the original enamel surface. This provides a tight chemical contact to the natural substrate utilizing the on-trend tissue engineering concept to regenerate rather than repair.

Recently, numerous in vitro attempts have been made to prepare enamel-like materials using biomimetic systems that contain nano-apatites or different organic materials used as analogues to organic matrix mainly in the form of slurries, solutions or pastes (26).

Inspired by the bio-mineralization process, chitosan hydrogel, in this study, is used as an organic template, due to its excellent biocompatibility, low toxicity and biodegradability 27). Also, the use of chitosan imparts the benefit of offering a protective effect against secondary caries due to its antibacterial properties along with preservation of enamel crystal orientation (28).

Chitosan was used in the form of a hydrogel as it was found by Min Han et al. (26) that hydrogels are the most versatile growth media for crystals, having an advantage over the solution system, since the initial formation of enamel apatite in nature occurs in a unique gel-like organic matrix and clinically, hydrogels are easier to handle.

In this study, the use of a phosphate ions dental adhesive agent formed of poly-anion organic molecules was beneficial for two different perspectives (29). 
Firstly, as explained by Kun Tian et al. (24) that phosphate ions on the dental adhesive agent should attract the positively charged-NH2 of the chitosan gels. Thereafter, the chitosan molecules should be oriented perpendicular to the substrate and parallel to each other. The functional carboxyl group that is present on the surfaces of chitosan, is effective for apatite nucleation, then apatite spontaneously forms on this surface in an ordered orientation.

The second perspective is that phosphate ions on dental adhesive agent can intake calcium and phosphorus from the artificial saliva solution, through the porous chitosan hydrogel, which contains plenty of water (up to $96 \mathrm{wt} \%$ ), providing space for calcium or phosphate ions to spontaneously diffuse into the lesion (27).

This study demonstrated that there is an evident precipitation of an enamel-like layer in both treatment groups (5 and 10 days incubation periods) using the chitosan biomimetic hydrogel, in contrast with the control group where the acid etch produced an irregular porous surface. Similar results were obtained by Kun Tian et al. (24), who have proposed the idea of using the chitosan hydrogel for enamel remineralization in a supersaturated solution. However, in the present study, using artificial saliva as a source of ions at $37^{\circ} \mathrm{C}$, mimicking biological conditions, was challenging, due to the limited ions concentration available for remineralization. Also, this concept was further advanced by exploring the effect of the biomimetic hydrogel within two different incubation periods.

SEM results revealed that nano-rod crystals have uniformly precipitated on the pre-existing enamel crystals in the 5 days incubated samples as well as the 10 days incubated samples. These small mineral structures had a potential to penetrate the lesion surface and enhance the remineralization along the whole lesion depth. However, the whole mineralization process seemed incomplete when studied for the 5 days incubation time in group II in comparison with the longer incubation period (10 days in group III), as the profile of acid-etched tooth enamel was still clearly visible when samples were observed after 5 days of treatments. While, samples of group III showed a superior and uniform re-establishment of surface integrity.

The most striking features of the current study were that the regenerated nano-rod crystals were deposited in a highly ordered manner and the long axes of the crystals were radially perpendicular to the underlying enamel surface. Bundles of parallel crystals formed enamel rod-like structures along their long axes as the chitosan hydrogel formed a three dimensional network, stabilizing $\mathrm{Ca}-\mathrm{P}$ clusters and guiding their arrangement into linear chains to restore the lesion.

These findings were in accordance with Ying Cao et al. (30) who effectively used an agarose model to regenerate an ordered enamel-like tissue.

In contrast with Ruan et al. (22) who developed an amelogenin-containing chitosan hydrogel for enamel reconstruction. Comparing the morphologies of the remineralized layers formed in the chitosan hydrogel with and without amelogenin, they observed that disordered structures with a porous morphology were formed without amelogenin, but ordered enamel-like structures were obtained in the presence of the protein.

Phosphorus and calcium are important for both skeleton and teeth. The normal $\mathrm{Ca} / \mathrm{P}$ molar ratio in enamel is 1.67
(31). With means of EDAX, we found that the mean $\mathrm{Ca} / \mathrm{P}$ ratio of control group was found to be 1.12, after the enamel reacted with etching agents, this could be explained by the fact that the content of $\mathrm{Ca} 2+$ on the etched enamel surfaces significantly lessened due to demineralization. On the other hand, the $\mathrm{Ca} / \mathrm{P}$ molar ratio for the enamel of the repaired groups had significantly improved. It measured a mean of 1.55 for group II indicating the formation of amorphous calcium phosphate phase. While the mean $\mathrm{Ca} / \mathrm{P}$ ratio for group III was 1.65 indicating the formation of a crystalline tooth-like apatite close to the stoichiometric $\mathrm{Ca} / \mathrm{P}$ ratio of the biological enamel.

An explanation of these interesting findings may be that chitosan acts as a template in the biomineralization process and controls the mineral crystallites through the molecular interaction between the polymer and minerals. The ion or cluster binding in the biomimetic mineralization system assembles into amorphous primary particles at the organic surface, and then the amorphous primary particles build up and form the oriented crystallization (32).

Chitosan not only provided a substrate to immobilize the nano-building units but also acted as a template for the structured assembly of the regenerated enamel layer.

\section{CONCLUSION}

The ultrastructural and elemental alterations noticed in this study substantiate the use of chitosan in case of enamel erosion. This model may be transferred in a straightforward manner to clinical use, but the mechanism needs further study. A strip containing the hydrogel and temporarily sealed on teeth to assist enamel reconstruction is envisioned.

\section{CONFLICT OF INTEREST}

The authors declare that they have no conflicts of interest.

\section{REFERENCES}

1. Cao Y, Mei ML, Li QL, Lo EC, Chu CH. Enamel prismlike tissue regeneration using enamel matrix derivative. J Dent. 2014;42:1535-42.

2. Dawes $\mathrm{C}$. What is the critical $\mathrm{pH}$ and why does a tooth dissolve in acid? J Can Dent Assoc. 2003;69:722-4.

3. Sudjalim TR, Woods MG, Manton DJ. Prevention of white spot lesions in orthodontic practice: a contemporary review. Aust Dent J. 2006;51:284-9.

4. Nobre-dos-Santos M, Rodrigues LK, Del-Bel-Cury AA, Cury JA. In situ effect of a dentifrice with low fluoride concentration and low $\mathrm{pH}$ on enamel remineralization and fluoride uptake. J Oral Sci. 2007;49:147-54.

5. Newby CS, Creeth JE, Rees GD, Schemehorn BR. Surface microhardness changes, enamel fluoride uptake, and fluoride availability from commercial toothpastes. J Clin Dent. 2006;17:94-9.

6. Young A, Thrane PS, Saxegaard E, Jonski G, Rölla G. Effect of stannous fluoride toothpaste on erosion-like lesions: an in vivo study. Eur J Oral Sci. 2006;114:180-3.

7. Magalhães AC, Stancari FH, Rios D, Buzalaf MA. Effect of an experimental $4 \%$ titanium tetrafluoride varnish on dental erosion by a soft drink. J Dent. 2007;35:858-61.

8. Skrtic D, Hailer AW, Takagi S, Antonucci JM, Eanes ED. Quantitative assessment of the efficacy of amorphous calcium phosphate/methacrylate composites in 
remineralizing caries-like lesions artificially produced in bovine enamel. J Dent Res. 1996;75:1679-86.

9. Reynolds EC, Cai F, Shen P, Walker GD. Retention in plaque and remineralization of enamel lesions by various forms of calcium in a mouth rinse or sugar-free chewing gum. J Dent Res. 2003;82:206-11.

10. Skrtic D, Eanes ED, Antonucci JM. Polymeric calcium phosphate composites with remineralization potential. Industrial Biotechnological Polymers 1995; 393-408.

11. Hannig M, Hannig C. Nanotechnology and its role in caries therapy. Adv Dent Res. 2012;24:53-7.

12. Li X, Pan D, Lin S, Zhuang Z, Linb Z. Facile in vitro hydroxyapatite remineralization of human enamel with remarkable hardness. Cryst Eng Comm. 2013;15:4351.

13. Bianco A, Cacciotti I, Lombardi M, Montanaro L, Gusmano G. Thermal Stability And Sintering Behaviour Of Hydroxyapatite Nanopowders. JTAC. 2007;88:237-43.

14. Wilcock CJ, Gentile P, Hatton PV, Miller CA. Rapid Mix Preparation of Bioinspired Nanoscale Hydroxyapatite for Biomedical Applications. J Vis Exp. 2017;(120).

15. Chen L, Yuan H, Tang B, Liang K, Li J. Biomimetic remineralization of human enamel in the presence of polyamidoamine dendrimers in vitro. Caries Res. 2015;49: 282-90.

16. Han M, Li QL, Cao Y, Fang H, Xia R, Zhang ZH. In vivo remineralization of dentin using an agarose hydrogel biomimetic mineralization system. Sci Rep. 2017;7: 41955.

17.Zikakis JP. Chitosan and Related Enzymes. Chitin, Orlando, Fla: Academic Press; 1984. p XVII.

18. Jay S, Dutta PK. Preparation, antibacterial and physicochemical behavior of chitosan/ofloxacin complexes. Int J Polym Mater. 2010;59:793-807.

19. Oyane A, Kawashita M, Nakanishi K, Kokubo T, Minoda $\mathrm{M}$, Miyamoto $\mathrm{T}$, et al. Bonelike apatite formation on ethylene-vinyl alcohol copolymer modified with silane coupling agent and calcium silicate solutions. Biomaterials. 2003;24:1729-35.

20. Yamaguchi I, Itoh S, Suzuki M, Osaka A, Tanaka J. The chitosan prepared from crab tendons: II. The chitosan/apatite composites and their application to nerve regeneration. Biomaterials. 2003;24:3285-92.

21. de Carvalho MMSG, Stamford TCM, dos Santos EP, Tenório P, Sampaio F. Chitosan as an oral antimicrobial agent. Science against microbial pathogens: communicating current research and technological advances A. Méndez-Vilas (Ed.) 2011. Available at : http://www.formatex.org/polymerscience1/chapters/542550.pdf

22. Ruan Q, Moradian-Oldak J. Development of Amelogeninchitosan Hydrogel for In Vitro Enamel Regrowth with a Dense Interface. J Vis Exp. 2014;51606.

23. Xu Z, Neoh KG, Lin CC, Kishen A. Biomimetic deposition of calcium phosphate minerals on the surface of partially demineralized dentine modified with phosphorylated chitosan. J Biomed Mater Res B Appl Biomater. 2011;98:150-9.

24. Tian K, Pengb M, Fei W, Ren XH. Induced Synthesis of Hydroxyapatite by Chitosan for Enamel Remineralization. Adv Mat Res. 2012:530:40-5.

25. Wang Y, Li B, Zhou Y, Jia D. In Situ Mineralization of Magnetite Nanoparticles in Chitosan Hydrogel. Nanoscale Res Lett. 2009;4:1041-6.
26. Han M, Li QL, Cao Y, Fang H, Xia R, Zhang ZH. In vivo remineralization of dentin using an agarose hydrogel biomimetic mineralization system. Sci Rep. 2017;7:41955.

27. Li B, Wang Y, Jia D, Zhou Y. Gradient structural bone-like apatite induced by chitosan hydrogel via ion assembly. J Biomater Sci Polym Ed. 2011;22:505-17.

28. Husain S, Al-Samadani KH, Najeeb S, Zafar MS, Khurshid Z, Zohaib S, et al. Chitosan Biomaterials for Current and Potential Dental Applications. Materials (Basel). 2017;10. pii: E602.

29. Das UM, G S. Bonding agents in pit and fissure sealants: a review. Int J Clin Pediatr Dent. 2009;2:1-6.

30. Cao Y, Mei ML, Li QL, Lo EC, Chu CH. Agarose hydrogel biomimetic mineralization model for the regeneration of enamel prismlike tissue. ACS Appl Mater Interfaces. 2014;6:410-20.

31. Wu X, Zhao X, Li Y, Yang T, Yan X, Wang K. In situ synthesis carbonated hydroxyapatite layers on enamel slices with acidic amino acids by a novel two-step method. Mater Sci Eng C Mater Biol Appl 2015;54:150-7.

32. Cao CY, Mei ML, Li QL, Lo EC, Chu CH. Methods for biomimetic remineralization of human dentine: A systematic review. Int J Mol Sci. 2015;16:4615-27. 